\title{
Post-COVID-19 paradoxical vocal fold movement disorder
}

\author{
Jérôme René Lechien ${ }^{1,2,3}$ (D) Marta P. Circiu ${ }^{1} \cdot$ Lise Crevier-Buchman ${ }^{1} \cdot$ Stephane Hans ${ }^{1}$
}

Received: 19 August 2020 / Accepted: 20 September 2020 / Published online: 30 September 2020

(c) Springer-Verlag GmbH Germany, part of Springer Nature 2020

\section{Dear Editor,}

A 28-year-old female presented to the laryngology office with a 7 -week history of sudden inspiratory noise and dyspnea. The patients suffered from COVID-19 at the end of March; the diagnosis being confirmed through nasopharyngeal swabs and RT-PCR. The COVID-19 related symptoms lasted 7 days and included loss of taste and smell senses, mild dysphonia, headache, and nasal obstruction. Patient did not have cough or fever. The overall clinical picture was mild to moderate and did not require hospitalization. At the exception of the loss of smell, the other symptoms completely resolved in one week. One week after the symptom resolution, she developed sudden inspiratory noise and dyspnea when speaking, both being persistent 7 weeks after the COVID-19 resolution. The disorder was present at rest, at both inspiration and expiration times and increased when patient was speaking for a long time. The patient did not report medical history and she did not practice sport. The general otolaryngological and neurological examinations were normal. Patient had no finding of laryngopharyngeal reflux and the reflux symptom score- 12 was 4 , making the

Stephane Hans, Lise Crevier-Buchman contributed equally to this work and are co-senior authors.

Electronic supplementary material The online version of this article (https://doi.org/10.1007/s00405-020-06391-z) contains supplementary material, which is available to authorized users.

Jérôme René Lechien

Jerome.lechien@umons.ac.be

1 Department of Otolaryngology and Head and Neck Surgery, Foch Hospital, Paris Saclay University, Paris, France

2 Department of Otolaryngology and Head and Neck Surgery, CHU de Bruxelles (Saint-Pierre), Université Libre de Bruxelles, Brussels, Belgium

3 Laboratory of Anatomy and Cell Biology, Faculty of Medicine, Research Institute for Health Sciences and Technology, University of Mons (UMONS), Avenue du Champ de Mars, 8, B7000 Mons, Belgium reflux diagnosis improbable. A videolaryngostroboscopy has been realized, reporting inspiratory and expiratory paradoxical movement of the vocal folds (Video 1). The best way to provoke the vocal fold paradoxical movement was the realization of a sustained vowel. The maximal phonation time was $3 \mathrm{~s}$. There were no additional laryngeal abnormalities. The laboratory testing did not find other infectious diseases (e.g., HZV, HSV, CMV, EBV, HBV, HCV, Influenza, HIV, Syphilis, Mycoplasma Pneumonia).

The patient did not report stress in its life over the past few months and had no apparent psychological disorder. A post-viral Paradoxical vocal fold movement disorder (PVFMD) diagnosis was retained and patient benefited from speech therapy. Four months after the onset of the disorder, the patient recovered after speech therapy. According to the patient clinical history, the development of PVFMD was attributed to the COVID-19.

PVFMD is a rare laryngeal disorder characterized by intermittent constriction of the vocal folds during respiration, stridor and dyspnea [1]. The cause of PVFMD is still unknown [1]. The disease development could be associated with emotional stress, laryngopharyngeal reflux, exercise or neurological nerve impairment [1]. Thus, some data support that the disorder may develop after viral-related laryngeal recurrent nerve impairment $[1,2]$. In this paper, we report the case of a young patient who developed PVFMD after COVID-19. The specific mechanism of PVFMD development in the present case remains unknown. The patient did not appear to have other common causes of PVFMD, including reflux, viral infection or psychological stress, which may support a potential association with COVID-19. The potential relationship between both conditions may be strengthened by the neuroinvasive potential of COVID-19 [3] and the vocal fold expression of ACE2 [4]. From a theoretical standpoint, the virus may spread into the tissue by the vocal fold ACE2 and may penetrate into the laryngeal nerve endings. The observation reported in this paper may be useful for otolaryngologists regarding the current pandemic. However, the association between COVID-19 and PVFMD will 
only be confirmed through future studies including similar patients without comorbidities associated with PVFMD.

Funding This study (response to the editor) has not received any support from funding agencies.

\section{Compliance with ethical standards}

Conflict of interest The author had no conflict of interest.

Ethical approval This article does not contain any studies with human participants or animals performed by any of the authors.

\section{References}

1. Koufman JA, Block C (2008) Differential diagnosis of paradoxical vocal fold movement. Am J Speech Lang Pathol 17(4):327-334. https://doi.org/10.1044/1058-0360(2008/07-0014
2. Hirano M, Tomita H, Nagashima T, Matsushita H, Kawasaki $\mathrm{H}$ (1970) Paradoxical movement of the vocal cord in recurrent laryngeal nerve paralysis. Nihon Jibiinkoka Gakkai Kaiho 73(7):885-891

3. Li YC, Bai WZ, Hashikawa T (2020) The neuroinvasive potential of SARS-CoV2 may play a role in the respiratory failure of COVID-19 patients. J Med Virol 92(6):552-555. https://doi. org/10.1002/jmv. 25728

4. Descamps G, Verset L, Trelcat A, Hopkins C, Lechien JR, Journe F, Saussez S (2020) ACE2 protein landscape in the head and neck region: the conundrum of SARS-CoV-2 infection. Biology (Basel) 9(8):E235. https://doi.org/10.3390/biology9080235

Publisher's Note Springer Nature remains neutral with regard to jurisdictional claims in published maps and institutional affiliations. 